\title{
Apuntes para pensar la articulación entre desarrollo territorial y educación a partir de la experiencia de una cátedra universitaria
}

\section{Notes to think about the articulation between territorial development and education from the experience of a university chair}

Denise Zenklusen ${ }^{1}$ y Mauricio Menardi ${ }^{2}$

Recibido 15/09/2020. Aceptado 15/02/2021

http://dx.doi.org/10.30972/dpd.10154819

\section{Resumen}

A partir de recuperar los aportes del enfoque del desarrollo territorial y de la pedagogía crítica, y tomando como punto de partida a la investigación-acción participativa, el presente artículo busca reflexionar sobre la experiencia de la enseñanza de la propuesta pedagógica de Aprendizaje-Servicio a estudiantes-docentes de la cátedra de Educación, desarrollo local y territorial de la Licenciatura en Educación de la Universidad Nacional de Rafaela.

Para ello, se propone, en primer lugar, reconstruir y sistematizar la experiencia de la cátedra durante el período 2018-2020. En segundo lugar, indagar sobre el uso del Aprendizaje-Servicio como una posibilidad pedagógica anclada en el territorio que complejiza el vínculo docente-estudiante y que presenta un rol diferente del estudiante en su proceso de enseñanza-aprendizaje. Y, finalmente, arrojar algunas pistas que inviten a pensar una educación en diálogo con el desarrollo y las transformaciones de los territorios.

Palabras clave: educación; desarrollo territorial; investigación acción.

\begin{abstract}
Starting from recovering the contributions of the territorial development approach and critical pedagogy and taking participatory action research as a starting point; This article seeks to reflect on the experience of teaching the Service-Learning pedagogical proposal to student-teachers of the Chair of Education, Local and Territorial Development of the Degree in Education at the National University of Rafaela.

It is proposed, first of all, to rebuild and systematize the experience of the chair during the period 2018-2020. Second, to inquire about the use of Service-Learning as a pedagogical

\footnotetext{
${ }^{1}$ Doctora en Antropología (UBA), Centro de Investigación y Transferencia de Rafaela (CONICET y UNRaf); Facultad de Cultura, Educación y Conocimiento (UNRaf) y Facultad de Trabajo Social (UNER). denisezenklusen@gmail.com

${ }^{2}$ Licenciado en Desarrollo Local (UNVM). UNRaf e Instituto PRAXIS, Facultad Regional Rafaela, UTN. mauricio.menardi@unraf.edu.ar
} 
possibility anchored in the territory that complicates the teacher-student bond and that presents a different role for the student in their teaching-learning process. And, finally, shed some clues that invite us to think about an education in dialogue with the development and transformation of the territories.

Keywords: education, territorial development, investigation action.

\section{Introducción}

La ciudad de Rafaela posee 92945 habitantes, según el Censo Nacional de 2010, y es el tercer centro urbano de la provincia de Santa Fe, detrás de Rosario y Santa Fe. Se ubica en la región centro-oeste de la provincia, siendo la cabecera del departamento Castellanos. Posee 540 industrias manufactureras, destacándose aquellas vinculadas a la metalmecánica (autopartes y equipos para la industria alimenticia) y a la producción de alimentos (lácteos y frigoríficos) (Icedel, 2018). Además, cuenta con un entramado institucional de 356 organizaciones de la sociedad civil (asociaciones vecinales, civiles, culturales, religiosas, fundaciones, cooperativas, mutuales, clubes, entre otras), 93 establecimientos educativos y 5 universidades (2 públicas y 3 privadas) y sedes de diversos institutos científicos y tecnológicos como el Inta, Inti y Conicet (Icedel, 2020). Según diversas investigaciones, la ciudad constituye un caso singular de desarrollo económico local en Argentina (Costamagna, 2015). Esta afirmación se sustenta, entre otras razones, en un proceso que comienza en la década de 1990, caracterizado por la conformación de un entramado institucional sólido y en la capacidad de las personas y de las diferentes organizaciones para constituir un ambiente dinámico con políticas activas. Costamagna (2015: 52) identifica cinco elementos comunes en este proceso: "el rol activo asumido por los gobiernos locales, la construcción de políticas desde el diálogo y la articulación, la integración de lo social y lo productivo, la mirada estratégica y una visión del desarrollo más allá del crecimiento". Estos elementos permiten comprender cómo se fortalece el desarrollo territorial en la ciudad.

En lo que respecta a la dimensión educativa, el Estado local, en su relación con las diferentes universidades, ha jugado un rol importante en la construcción de aprendizajes y capacidades colectivas para el desarrollo territorial (Costamagna y Menardi, 2019). En este escenario, marcado por una impronta basada en el desarrollo territorial y en la búsqueda de promover el desarrollo de las capacidades de las personas y de las organizaciones para dar respuestas a las problemáticas, es en 2014 en que se crea la Universidad Nacional de Rafaela (UNRaf), a partir de la Ley $\mathrm{N}^{\circ}$ 27062. Ubicada en la ciudad homónima, es la tercera universidad nacional de la provincia de Santa Fe, luego de la Universidad Nacional del Litoral (1919) y de la Universidad Nacional de Rosario (1968). En su proyecto inicial se establecen 
los ejes fundamentales que consolidan su identidad institucional: la innovación, la competitividad y el desarrollo ligado al territorio, así como también el vínculo y la articulación con la comunidad.

El artículo $N^{\circ} 5$ del Estatuto provisorio define que es misión de la UNRaf constituirse en un espacio institucional que contribuya a fortalecer el sistema educativo, científico y tecnológico en todos sus niveles y donde se "formen personas altamente calificadas y comprometidas, capaces de diseñar y conducir las estrategias del sector productivo laboral, anticipar los desafíos de la gestión territorial y ambiental y consolidar la cultura de la cooperación, la igualdad y la responsabilidad pública".

Por su parte, la oferta educativa de las diferentes carreras que ofrece la universidad contempla estos ejes. Cada una de ellas propone un plan de estudio donde las materias dialogan con la misión de la institución y establecen una articulación con los territorios. En este contexto, y frente a la demanda en la ciudad y la región ${ }^{3}$, es que en 2017 se crea el ciclo de complementación curricular de la Licenciatura en Educación, dirigido a profesores y profesoras egresadas de instituciones universitarias o superiores no universitarias con títulos en especialidades pertenecientes al campo de la educación y de la enseñanza. Una de las particularidades que reviste la licenciatura -al menos durante el período 2017-2020- es que gran parte del estudiantado ejerce como docente en Nivel Primario, Secundario y/o Terciario de escuelas e institutos terciarios públicos y privados de la ciudad de Rafaela y la región. Por ello, es que a lo largo de este escrito utilizaremos la categoría estudiante-docente para referirnos a quienes formaron parte de la cátedra. Puntualmente, la materia Educación, desarrollo local y territorial compone el segundo año de la licenciatura y tiene como objetivos: 1. Abordar la temática del Desarrollo Territorial (DT) integrando los aportes teóricos de diversas disciplinas comprometidas en dichas cuestiones; 2 . Realizar un proceso de enseñanza-aprendizaje basado en un enfoque multidisciplinar, formando conocimientos bajo una lógica integradora de "pensamiento complejo", aplicable al análisis y prácticas de desarrollo territorial; 3. Reflexionar sobre el papel de la educación en las estrategias de desarrollo territorial y en la construcción de capacidades para el mismo; 4. Abordar el enfoque pedagógico para el desarrollo territorial como estrategia de intervención en los territorios y; 5 . Reflexionar críticamente sobre los procesos de DT y favorecer las actividades de investigación y desarrollo.

En 2018 iniciamos su dictado con una propuesta que buscaba articular los diálogos entre el enfoque del desarrollo territorial y la pedagogía crítica. Al mismo tiempo, y en el ejercicio práctico de la materia, propusimos el desarrollo de proyectos de Aprendizaje-Servicios (en

\footnotetext{
${ }^{3}$ La ciudad de Rafaela y su entorno regional comprenden una población que supera los 520000 habitantes y más de 160 localidades de mediana y pequeña escala. El área de influencia proyectada de la UNRaf abarca el norte y oeste de Santa Fe, y las zonas limítrofes de las provincias de Córdoba y Santiago del Estero.
} 
adelante ApS) como un enfoque pedagógico que contribuía a la apropiación de los contenidos de la materia y que les ofrecía herramientas a los/as estudiantes-docentes para reflexionar sobre el ejercicio de su práctica docente en articulación con las demandas y problemáticas en los territorios.

Luego de tres años de dictado de la materia y en la búsqueda de problematizar nuestra práctica docente en diálogo con la de los y las docentes-estudiantes ${ }^{4}$, en este artículo proponemos reflexionar sobre la experiencia de la enseñanza de la metodología Aprendizaje-Servicio a estudiantes-docentes de la cátedra de Educación, desarrollo local y territorial de la Universidad Nacional de Rafaela durante el período 2018-2020. En articulación con los aportes del enfoque del desarrollo territorial y de la pedagogía crítica, partimos del supuesto teórico, pero también político, de que la formación de educadores y educadoras se debe dar a través de una práctica reflexiva. Entendemos que la enseñanza del ApS es también una manera de contribuir a la responsabilidad social universitaria de formar sujetos capaces de dar respuestas a las demandas de los territorios. Por consiguiente, en un primer apartado, presentamos la propuesta teórica-metodológica desde donde miramos y nos posicionamos para realizar y analizar la experiencia. Así, recuperamos los aportes del enfoque del desarrollo territorial y los aportes de la pedagogía crítica en cruce con una metodología de investigación-acción participativa en la cual nos reconocemos, junto con nuestros/as estudiantes-docentes, como educadores/as. En un segundo apartado, y a partir de sistematizar la experiencia, retomamos dos dimensiones que emergieron durante el dictado de la materia. Por un lado, en nuestra práctica de docentes universitarios nos encontramos con el desconocimiento -por parte de estudiantes-docentes-del vínculo entre desarrollo territorial y educación. Y, al mismo tiempo, y por otro lado, con la dificultad de pensar a las instituciones educativas como parte de los territorios. Para problematizar esto, desde la cátedra llevamos a cabo una serie de estrategias sobre las cuales nos interesa volver. Un segundo apartado, donde reflexionamos sobre los proyectos de AprendizajeServicio como alternativa a otras formas de enseñanza-aprendizaje. Finalmente, desandamos la importancia de enseñar e incorporar en la práctica docente -tanto universitaria como de los otros niveles- metodologías que recuperen el rol activo de los y las estudiantes en los procesos de aprendizaje y donde las aulas se constituyan en espacios de cambio social en articulación con los territorios. En este sentido, enmarcamos este trabajo en la necesidad de construir y enseñar metodologías -y por qué no una pedagogía- comprometida con las demandas de los territorios.

\footnotetext{
${ }^{4}$ En la búsqueda por problematizar nuestra práctica docente, queremos realizar un agradecimiento especial a los y las integrantes del Instituto Praxis (UTN-FRRa), quienes acompañaron con diálogos, intercambios y lecturas este proceso.
} 


\section{Herramientas teóricas-metodológicas}

Un primer aporte que sumamos en nuestra cátedra es el enfoque del desarrollo territorial. Tomando los trabajos de diversos autores (Alburquerque, Costamagna y Ferraro, 2008; Arocena, 2013; Coraggio, 2004; Madoery, 2006; Villar 2007), podemos definir el desarrollo territorial como un proceso político de fortalecimiento y construcción de capacidades en una sociedad que habita un territorio determinado para mejorar el bienestar de sus ciudadanos y de las generaciones futuras.

Para una mejor comprensión del enfoque, es importante que nos detengamos en algunas categorías presentes en el concepto. En primer lugar, destacamos la idea del desarrollo como un proceso, ya que no se trata de algo estático o de un estadio final, sino de algo que está en permanente construcción a lo largo del tiempo. $Y$ decimos que es político porque dicho proceso es pensado, planeado, gestionado y promovido por actores locales. Este proceso político va configurando los territorios a través del tiempo, generando sus propias improntas y características identitarias, culturales, políticas, económicas y ambientales. Por ello, podemos decir que todos los territorios son diferentes. Pero para que un proceso se configure como uno de desarrollo territorial es importante que busque fortalecer y ampliar las capacidades de los actores locales para definir las problemáticas que los aquejan y construir juntos las posibles soluciones. Esto se debe realizar a través de la participación, el diálogo y el aprendizaje colectivo de los actores locales. El objetivo último de todo proceso de desarrollo territorial debe ser la satisfacción de las necesidades humanas, desde aquellas más básicas y fundamentales, como alimentación, salud, vivienda y educación, hasta las de protección, entendimiento, participación, ocio-recreación, creación, identidad, libertad y trascendencia (Elizalde, Max-Neef y Hopenhayn, 1986). Todo esto en un marco de sostenibilidad necesario para asegurar la vida de las generaciones futuras, donde el modelo socioeconómico esté en armonía con el medio natural en el cual se encuentra inserto.

Es en el marco de este enfoque de desarrollo territorial que desde hace una década investigadores e investigadoras de la Red DETE, ConectaDEL y del Instituto Praxis (UTN FRRa) vienen reflexionando y promoviendo una praxis del desarrollo territorial, denominada Enfoque Pedagógico para el Desarrollo Territorial (EPDT). Dicho enfoque propone una forma de entender y actuar en la construcción de procesos de aprendizajes para el cambio en el territorio de forma coherente con una construcción social y política. El EPDT supone un modo de comprender la vinculación teoría-práctica, el reconocimiento del otro (saberes locales, prácticas y experiencias), la vinculación y construcción basada en el diálogo y la resolución de conflictos promoviendo instancias democráticas (Costamagna, 2020). 
EI EPDT promueve una mirada distinta de plantear la relación educador/educando/territorio, fomentando una construcción de conocimientos con mayor democracia y orientados a la transformación. Algunos de los elementos centrales del EPDT son: a) la evolución de la formación tradicional al concepto de praxis (acción-reflexiónacción); b) la construcción de capacidades en el desarrollo territorial como objetivo; c) la no neutralidad del formador; d) plantea como un eje central el proceso de cogeneración de conocimientos; e) busca integrar la relación con el entorno de los procesos formativos (y de investigación) y la importancia del contexto en el desarrollo territorial; y f) la comunicación y sistematización de los procesos como estrategias e instrumentos del enfoque (Costamagna, 2015).

El EPDT retoma la propuesta de la pedagogía crítica latinoamericana buscando poner en foco el fortalecimiento y la construcción de capacidades para el desarrollo territorial. En esta línea, y en diálogo con las reflexiones de Freire ([1965] 2008), entendemos a la educación como una práctica de la libertad, en la cual es importante resaltar el carácter político y ético del problema educativo. Desde la propuesta pedagógica de Freire ([1965] 2008), los nuevos procesos formativos deben generar un "hombre nuevo", consiente de su realidad y comprometido con su transformación, resaltando la construcción dialógica, el humanismo crítico, las prácticas emancipadoras y la reinvención como aspectos fundamentales para la constitución de vínculos sociales solidarios. En esta línea, Freire (1989) formula dos grandes modelos educativos que solo pueden aprehenderse cabalmente en su mutua oposición. Por un lado, el modelo bancario que supone la existencia de un educador o educadora que "lo sabe todo" y de un/a estudiante que "lo ignora todo". La enseñanza se define como depósito, transmisión e instrucción mecánica. En contraposición, en el modelo liberador, la tarea educativa se sustenta en la idea de que tanto educador/a como educando/a saben y aprenden a la vez que enseñan. La enseñanza se entiende como una actividad problematizadora, crítica e investigativa que tiene por objeto desvelar la realidad para poder situarnos. En esta propuesta, el/la educador/a debe asumir una profunda coherencia entre el discurso que pronuncia y la práctica que desarrolla. Así, el/la educador/a se mueve "entre varios polos o tensiones: la palabra y el silencio; la práctica y la teoría; y el aquí y ahora suyos en relación con el aquí y el ahora de los educandos/as" (Monclús, 1988: 131). La relación educativa es una relación de diálogo y de apertura, basada en la autonomía y la libertad tanto del educador/a como de los educandos, en la línea del pensamiento existencialista tan apreciado por el pedagogo brasileño. El educador, señala Freire, tiene que ser un/a inventor/a y un reinventor/a constante "de todos aquellos medios y todos aquellos caminos que faciliten más y más la problematización del objeto que ha de ser descubierto y finalmente aprehendido por los educandos" (Freire, 2000: 18). En esta propuesta, el 
educador o la educadora rechazan los valores dominantes que se imponen a la escuela, porque su aspiración debe ser la transformación del statu quo (Freire y Macedo, 1987).

En diálogo con el enfoque territorial y los aportes de pedagogía crítica es que partimos, junto con Tapia (2008a), de reconocer a la universidad como "parte" del conjunto de la comunidad, ni aislada ni supeditada a las demandas del "afuera". Entendemos que el territorio no puede ser visto como mero destinatario, sino como un espacio donde se aprende, se investiga, se construye con otros actores y donde las iniciativas "Ilevadas a cabo junto con la comunidad son para estudiantes y docentes también una forma de aprender y de investigar" (Tapia, 2008b: 36). Y encontramos en la apuesta del Aprendizaje-Servicio algunos hilos para trenzar.

En las últimas décadas, el ApS se extendió rápidamente en diferentes países a nivel mundial. Alrededor de 2005, comenzó en Estados Unidos y en Argentina (Escofet et al. 2016), y a partir de ahí en diferentes países fueron surgiendo de manera simultánea iniciativas similares. Como señala Annete (2005), todas estas experiencias tienen un elemento en común: el ApS supone un medio para promover la participación de jóvenes, niños y niñas al servicio de la comunidad, tanto en la enseñanza obligatoria como en la educación superior. A su vez, favorece la articulación de dimensiones clave en las instituciones educativas: teoría y práctica; aula y "realidad"; instituciones educativas y comunidad (Manzano, 2010; Rodríguez, 2014). Por ello, para que una práctica de ApS se considere como tal, debe atender al aprendizaje académico, al diálogo horizontal con el territorio y al rol protagónico del estudiantado. Las investigaciones sobre ApS son variadas y responden a diferentes enfoques. Así, algunos y algunas autores/as lo definen como una estrategia metodológica (Bednarz et al., 2008; Reinders, 2010) o como una metodología en sí misma (Furco, 2003; Puig, 2009; Rodríguez, 2014; Tapia, 2008a). Otros autores y autoras lo entienden como una propuesta pedagógica (Cecchi, 2006) y, finalmente, están aquellos/as que lo definen como un programa, una pedagogía y una filosofía (Puig, Battle, Bosch y Palos, 2007). Todos estos enfoques coinciden en que el ApS constituye una manera de adquirir aprendizajes mediante la práctica. Es decir, dándoles sentido y significado en cuanto se contextualiza su utilidad (Escofet et al., 2016).

Al mismo tiempo, desde el ApS, la institución educativa no solo se convierte en un espacio de diálogo, sino que "entra en diálogo" y en articulación con el territorio conformando un modo relacional, recíproco, con intereses y corresponsabilidades definidas. En este sentido, en la articulación con el enfoque del desarrollo territorial y retomando los aportes de Rodríguez (2013: 98), consideramos que el modelo formativo de las universidades públicas en Argentina debe enfocarse en una línea que promueva en su práctica, pero también en sus espacios de convivencia y trabajo, situaciones de implicación con los territorios. Como señalan Folgueiras y Martínez (2009), estaríamos educando a estudiantes 
hacia el desarrollo de competencias vinculadas a cuestiones sociales y éticas; basadas en un aprendizaje significativo, colaborativo y dialógico (Arandia, Alonso y Martínez, 2010). Encontramos en el ApS algunas líneas que se trenzan con la propuesta de la pedagogía crítica y que al mismo tiempo "provoca efectos en el desarrollo personal, cambios en las instituciones educativas y sociales que lo impulsan, y mejoras en el entorno comunitario que recibe el servicio" (Puig y Palos, 2006: 63).

Hasta aquí hemos explicitado el abordaje teórico desde el cual nos interesa enmarcar este trabajo. Tal como lo venimos señalando, entendemos que en el proceso de identificar las dinámicas, los tiempos y recorridos de un espacio -en nuestro caso, los tres años consecutivos de la cátedra de Educación, desarrollo local y territorial y de la enseñanza del ApS a estudiantes-docentes- podemos recuperar saberes de esa práctica docente. Para ello, consideramos en términos metodológicos indispensable retomar la propuesta de sistematización de experiencia que, siguiendo a Jara (1994), podemos definirla como aquella interpretación crítica de una o varias experiencias que, "a partir de su ordenamiento y reconstrucción, descubre o explicita la lógica del proceso vivido, los factores que han intervenido en dicho proceso, cómo se han relacionado entre sí, y por qué lo han hecho de ese modo" (Jara, 1994: 22). La interpretación de esa experiencia es clave para recuperar aprendizajes que posean utilidad para el futuro y mejorar la calidad de los procesos.

En la búsqueda de construir conocimiento sobre la articulación entre desarrollo territorial, educación y ApS, es que en términos metodológicos decidimos recuperar la investigaciónacción participativa como una metodología que nos permitía problematizar nuestra práctica docente, pero también el espacio que fuimos construyendo junto con los y las estudiantesdocentes. Partimos del supuesto de entender a la enseñanza como un proceso de investigación y de continua búsqueda. Esto conlleva abordar el trabajo docente, integrando la reflexión y el trabajo intelectual en el análisis de las experiencias que se realizan, como un elemento esencial de lo que constituye la propia actividad educativa. En la concepción de los métodos y técnicas participativas prima la idea de un aprendizaje activo, de creación y recreación del conocimiento por los y las estudiantes mediante la actividad sistematizada. Recuperando los aportes de Latorre (2007), entendemos a la investigación-acción participativa como un método para mejorar y/o transformar la práctica social y/o educativa, al mismo tiempo que busca mejorar la comprensión de dicha práctica y articular, de manera permanente, la investigación, la acción y la formación. Para ello, es central la participación de los y las docentes y también de los y las estudiantes-docentes.

Los problemas guían la acción, pero lo fundamental en la investigación-acción participativa es la exploración reflexiva que el/la profesional hace de su práctica, no tanto por su contribución a la resolución de problemas, como por su capacidad para que cada profesional reflexione sobre su propia práctica, la planifique y sea capaz de introducir 
mejoras progresivas. En este marco teórico-metodológico es que proponemos sistematizar la experiencia de la cátedra Educación, desarrollo local y territorial. Para ello, recuperamos los registros que documentamos como cátedra entre 2018 y 2020 . Estos registros se componen de cuadernos de apuntes personales o diarios de campo, diseño de actividades, listas de participantes, informes y reportes, cuestionarios online, reflexiones individuales y colectivas, proyectos de ApS, comentarios en el campus virtual de la materia. Además, no solo proponemos registros escritos, sino también fotografías, afiches, dibujos y mapas. Todo este material forma parte del corpus empírico construido durante el dictado de la materia y es a partir del cual decidimos realizar la sistematización en pos de reflexionar no solo sobre nuestras prácticas docentes, sino de generar aportes para abonar y nutrir la articulación entre desarrollo territorial y educación.

\section{El aula como espacio de encuentro entre estudiantes-docentes, docentes y el territorio}

Como mencionamos en un comienzo, la Licenciatura en Educación tiene la particularidad de que se conforma como un ciclo complementario para aquellas personas que ya cuentan con un título terciario o de profesorado universitario. Por ello, al menos en este primer período que buscamos analizar, el estudiantado se compone por docentes quienes en su mayoría se encuentran ejerciendo la docencia en diferentes instituciones educativas de Nivel Terciario, Secundario, Primario e Inicial. En este sentido, y como primer desafío de la cátedra, nos vimos en la necesidad de reacomodar los contenidos a la luz de las características de los diferentes cursos. Así, emergía con fuerza -durante las presentaciones y los diálogos- la necesidad de reflexionar sobre su propia práctica docente en sus diferentes instituciones educativas. En segundo lugar, nos encontramos con el desconocimiento por parte de los y las estudiantes-docentes sobre el enfoque de desarrollo territorial. Inmediatamente, asociaban la idea de desarrollo al concepto de economía y al mismo tiempo con la dificultad de pensar el aula -y más precisamente las instituciones educativas- como un actor más dentro del territorio.

Ante este nuevo contexto que surgía durante las clases, nos propusimos realizar dos movimientos durante 2018 y 2020 de nuestro ejercicio docente. El primer movimiento consistió en construir colectivamente una mirada del desarrollo territorial en articulación con la educación. El segundo de ellos consistió en incorporar la reflexión continua de su experiencia docente como parte central del desarrollo de los contenidos. Para poder llevar a cabo estos dos movimientos, recuperamos la propuesta de Costamagna (2015) y con ella una serie de estrategias del enfoque pedagógico para el desarrollo territorial. De esta manera, propusimos construir el espacio del aula como un espacio de diálogo desde la formación. Entendemos a los espacios de diálogo como los "lugares formales e informales 
que actúan como nexo del proceso de construcciones compartidas" (Costamagna, 2015: 109). Y, en esta línea, Peiretti y Spinelli (2016) refuerzan la necesidad de motivar la investigación por parte de los y las estudiantes a partir del estudio de casos. Esto revaloriza el concepto de praxis, según el cual se retroalimentan teoría y práctica y donde los procesos de formación no ocurren solo en espacios cerrados, sino que también se dan en la interacción de diferentes actores.

A lo largo de las clases invitamos a diferentes actores del territorio a que dialoguen junto con los y las estudiantes-docentes sobre experiencias del desarrollo territorial. Durante el primer año, trabajamos, fundamentalmente, con experiencias de articulación del municipio de la ciudad de Rafaela con otros actores del territorio: "además de conocer lo que sucede en la ciudad, se pudo visualizar con ejemplos concretos algunas de las posturas que se proponen desde los textos y autores trabajados" (Estudiante-docente, comunicación personal, 2018); "nos permitió conocer el territorio a partir de cada charla de los invitados", (Estudiante-docente, comunicación personal, 2018), fueron algunas de las palabras de los y las estudiantes. En 2019, y a partir de los diálogos con los y las estudiantes-docentes de 2018, decidimos priorizar experiencias vinculadas a procesos educativos. Para ello, no solo reconstruimos experiencias de educación formal -de la universidad y de las escuelas-, sino también de experiencias no formales. Consolidar estos espacios facilitó la apropiación del enfoque de desarrollo territorial desde la educación.

Algunos de los comentarios fueron: "las charlas sirvieron para poder conocer sobre ciertas actividades que desconocía, además de relacionarse con la teoría trabajada en las diferentes clases" (Estudiante-docente, comunicación personal, 2019); "algunos invitados con aportes que me resultaron más valiosos y otros no tanto, pero considero una buena opción la de contar con miradas de diversos campos" (Estudiante-docente, comunicación personal, 2019) y "sin dudas es una técnica muy acertada de mostrar y visualizar lo trabajado en clases a partir de la visión de territorio que cada invitado trae consigo a cada una de las clases, se torna muy práctico y eficaz poder visualizar lo que sé desde la cátedra" (Estudiante-docente, comunicación personal, 2019). Estos registros plasman la importancia de proponer espacios de diálogos en las aulas y con otros actores, no necesariamente docentes. En los discursos de los y las estudiantes-docentes aparece con fuerza la división histórica que solemos hacer desde la enseñanza universitaria entre teoría y práctica. La apuesta de trabajar con invitados e invitadas -con el "estudio de caso" - de alguna manera pretendió problematizar esta dicotomía y proponer una instancia dialógica de construcción y apropiación del conocimiento.

En estos encuentros con actores del territorio se produjo el segundo movimiento que consistió en comenzar a pensar la noción de educación desde un enfoque que trascienda el espacio del aula, de allí la estrecha articulación que planteamos con el desarrollo territorial y 
con los aportes de la pedagogía crítica. Durante las diferentes cursadas, nos encontramos con una mirada, por parte de los y las estudiantes-docentes, de la educación anclada al espacio institucional y, específicamente, áulico. Y esto en parte se comprende atendiendo a la formación, a sus lecturas, pero especialmente a sus trayectorias como docentes y con las propias lógicas que revisten las instituciones educativas y que se encuentran cotidianamente. Neirotti (2005) sostiene que lo que distingue a la escuela como institución es la relación pedagógica que se construye allí. Es en el espacio del aula donde se establece el vínculo entre docentes y estudiantes. Sin embargo, y al mismo tiempo, las instituciones educativas se encuentran ubicadas en espacios geográficos diferentes y cuentan con estudiantes que provienen de medios socioeconómicos y culturales diversos. En este sentido, el autor nos arroja algunas pistas para pensar este contexto y entiende que es necesario trabajar hacia una mayor regionalización e incluso "localización" del currículo (Neirotti, 2005).

En esta línea, Neirotti (2005) propone como desafío la necesidad de generar un mayor acercamiento entre la escuela y la comunidad. Frente a este planteo y ante lo que se nos presentaba en el aula, es que decidimos combinar la presentación de experiencias de actores del territorio como una primera estrategia para incorporar y problematizar colectivamente la categoría de territorio y de actores en articulación con la educación. Y, como señala una de las estudiantes-docentes, "la materia me ofreció la posibilidad de acercarme a un campo teórico y práctico, relacionado con el desarrollo territorial, que no conocía, y me ayudó a pensar en las posibilidades de extender la propuesta de mis materias al entorno, involucrando a la escuela con otras instituciones y enriqueciendo el trabajo en conjunto" (estudiante-docente, comunicación personal, 2020). Esto fue posible a partir de establecer como objetivo de la materia la construcción de un proyecto de $\mathrm{ApS}$, como una manera de comenzar a incorporar el enfoque del desarrollo territorial. A continuación, nos interesa reflexionar sobre ese proceso.

\section{Construyendo una alternativa educativa: los proyectos de Aprendizaje-Servicio}

Como mencionamos en el apartado anterior, nos posicionamos a partir de dos movimientos: pensar la educación en articulación con el desarrollo territorial, para luego reflexionar y proponer una educación por fuera del aula y en diálogo con los territorios. En estos dos movimientos es que realizamos una apuesta a incorporar la elaboración de proyectos de Aprendizaje-Servicio como una metodología que articula una propuesta educativa con el desarrollo de los territorios. Fue así que nos encontramos con un desconocimiento por parte de los y las estudiantes-docentes de los proyectos de ApS o, en algunos casos, realizaban estos proyectos sin enmarcarlos en esta propuesta. 
Quizás son miradas o formas que en las escuelas se sostienen, o intentan aparecer, pero no en este formato. La estructura del proyecto de aprendizaje en servicio le otorga forma y estructura a lo que intentamos hacer a veces desde las instituciones. (Estudiante-docente, comunicación personal, 2018)

El proyecto es interesante no solo para pensarlo y trabajarlo con nosotros, sino para poder replicar la experiencia en las aulas con nuestros propios alumnos. Seguramente con más tiempo de cursado podríamos profundizar y ponerlo en práctica. (Estudiante-docente, comunicación personal, 2019).

Nos permite pensar en las diferentes formas de abordar la práctica docente, posicionándonos desde un lugar de facilitador, porque creo que los alumnos aprenden si hay sentimientos y acciones prácticas. (Estudiante-docente, comunicación personal, 2018)

Tal como señalamos en el apartado teórico-metodológico, entendemos al ApS como una alternativa a otros modos de enseñanza-aprendizaje, donde los y las protagonistas son los y las estudiantes, y donde la articulación entre la escuela y los diferentes actores sociales del territorio resulta central, en pos de contribuir a la transformación de una realidad. De esta manera, a lo largo de las diferentes clases trabajamos en la elaboración de proyectos de ApS que surgieron al calor de sus preocupaciones, tomando como punto de partida sus propias instituciones.

Las temáticas de los proyectos fueron variadas, pero siempre en articulación con las materias que dictaban y los contenidos que desarrollaban en sus propias clases. Así, y a partir de problemáticas cercanas a cada institución educativa, se propusieron proyectos vinculados a lo ambiental, a la seguridad vial, al consumo sustentable de alimentos, a los vínculos intergeneracionales, al uso de tecnología, a la ciudadanía y los derechos, entre otros. Si bien algunos proyectos reconstruyeron las problemáticas en interacción con los y las estudiantes, dado que la materia se desarrolla en el transcurso de 7 clases, nos topamos con la dificultad de que la mayor parte de los y las estudiantes-docentes pudieran implementar metodologías participativas para la construcción de las problemáticas a trabajar.

En este escenario, recurrimos a otras estrategias en pos de garantizar el pilar del ApS: la centralidad de los niños, las niñas y los y las jóvenes. De esta manera, y a partir de diagnósticos -en algunos casos participativos- que realizaron los y las estudiantes-docentes, algunos trabajos recuperaron experiencias de años anteriores que podrían transformarse en proyectos de ApS; otros establecieron diálogos con sus estudiantes y con el cuerpo docente de sus instituciones para orientar los proyectos $y$, finalmente, otros proyectos priorizaron las preocupaciones que emergen cotidianamente en las aulas junto con sus estudiantes.

La escuela como institución social, es decir, como una entidad inserta en el entramado de instituciones sociales que componen un territorio y una comunidad. Esto implica aceptar que 
la escuela está siendo interpelada por problemáticas sociales que impactan en su cotidianeidad, y también entender que se constituye como un núcleo fundamental para la construcción de redes que aborden los diversos problemas sociales de forma colectiva. (Estudiante-docente, comunicación personal, 2019)

Los procesos de educación vinculados al desarrollo territorial se constituyen en nuevos aprendizajes para la comunidad. Así, la finalidad de la educación vinculada al desarrollo comunitario -al desarrollo de las personas y los colectivos- resume la perspectiva desde la que pensamos el proyecto en salida. (Estudiante-docente, comunicación personal, 2020)

Una escuela de "puertas, ventanas y techos" abiertos a la sociedad en su conjunto, que no espera recetas mágicas, que no tiene todas las respuestas, pero que se apoya en redes y alianzas con otros actores e instituciones y que confía en el cambio y en la potencialidad de la comunidad. (Estudiante-docente, comunicación personal, 2019).

Estas expresiones, que emergieron durante el cursado de la materia, permiten reflexionar sobre la ausencia que hay en la práctica docente de incorporar metodologías que pongan a los y las estudiantes como protagonistas del proceso de aprendizaje, que articulen con otros actores sociales y en donde el o la docente se conviertan en un facilitador pedagógico. Acompañando el desarrollo de estos proyectos, desde la cátedra y desde los aportes del desarrollo territorial, propusimos trabajar con el rol de el/la facilitador/a. A partir de recuperar los aportes de Costamagna y Larrea (2017: 81), entendemos que en los procesos de desarrollo territorial ningún actor puede tomar las decisiones por otro y, por lo tanto, la estrategia más viable para "liderar un proceso de DT es facilitarlo, es decir, generar las condiciones para que otros actores reflexionen, decidan y actúen". Así, la facilitación se refuerza como estrategia para situaciones de complejidad, y los actores facilitadores son los y las encargados/as de dicha estrategia. En este sentido, presentamos durante el cursado abordar el rol de el/la docente en tanto un facilitador/a de los procesos educativos, y en el caso de los proyectos ApS, el responsable de guiarlo. En la práctica no solo lo planteamos en términos teóricos, sino que, a su vez, llevamos a cabo diferentes estrategias pedagógicas lúdicas y participativas para que el rol de facilitador/a se haga experiencia. Para ello, planificamos dos actividades: una de escritura y reflexión sobre su rol en las instituciones y la otra lúdica, donde a través de un juego revisamos el lugar de los actores sociales, de el/la facilitador/a y la búsqueda de trabajar colectivamente en pos de un objetivo.

En este sentido, como docentes partimos desde una postura que entiende el proyecto A$\mathrm{P}$ con una orientación transformadora y emancipadora. En el cual, no solo se trata de asistir una demanda concreta, sino de generar acciones pensadas de manera bidireccional, teniendo en cuenta lo pedagógico y lo comunitario. (Estudiante-docente, comunicación personal, 2020) 
Cómo uno puede mirarse desde los territorios, la apertura hacia el afuera. Sea un ida y vuelta. Me llevó a eso, a repensar esas cosas, a ver al educador como parte de los territorios. (Estudiante-docente, comunicación personal, 2020)

Empezar a pensarnos como actores territoriales, qué proyectos, qué orientación socioeducativa. (Estudiante-docente, comunicación personal, 2020).

Desde la perspectiva del ApS, la institución educativa no solo se convierte en un espacio de "diálogo", sino que "entra en diálogo" con la comunidad, conformando un modo relacional, recíproco, con intereses y corresponsabilidades definidas. A través de los proyectos de ApS, la institución se transforma en un espacio interno de participación, de creación de democracia y desarrollo, de visión común y búsqueda de una línea de actuación por "aprender y actuar juntos". Y es un espacio que aúna y articula esfuerzos con otras instituciones y actores sociales del territorio, que integra objetivos curriculares y necesidades, y que plantea un trabajo coordinado que fortalece el tejido social y contribuye a la creación de capital social. En esta línea, el/la docente ocupa un lugar central en tanto es la persona encargada de facilitar -de motozirar- ese proceso.

Puiggrós y Gómez Sollano (2003) sostienen que una "alternativa" responde a una inquietud de búsqueda, a una inconformidad frente a una situación dada, de un anhelo por crear mejores condiciones de vida; una alternativa es algo más que una creación para el plazo corto o mediano, más bien responde a la idea de proyecto de largo alcance, a la creación de propuestas con creatividad que se concretan a través de programas que apuntalan ciertas opciones de solución. Los proyectos alternativos generan significaciones compartidas que parten de necesidades (lo que es y no puede ser de otra manera), de experiencias (en sus múltiples acepciones: identitaria, performativa o como legado) y de expectativas en tanto inéditos posibles (utópicos o distópicos).

Las alternativas se definen en el marco de las prácticas sociales, de las luchas hegemónicas, en los diferentes niveles y planos de lo social, en la microsituación o en un sector específico como el económico o el político. Se vincula con el trabajo de construir la realidad como campo problemático (Zemelman, 1987), de modo que pueda ser abordada como objeto de conocimiento. En este sentido, "alternativas" es parte de un instrumental que hace posible el reconocimiento de hechos, problemáticas, actores que no serían visibles sin esa construcción categorial.

De este modo, tiene como función deconstruir el discurso establecido, por lo que permite poner en evidencia la lógica de configuración de la hegemonía. De ahí que concebir a la educación como práctica formadora de sujetos, cuyo soporte institucional es variado, permite abrir la mirada a una multiplicidad de prácticas no reconocidas como pedagógicas, a veces ni siquiera por los propios actores que las llevan adelante, pero que tienen eficacia histórica o capacidad de intervención, lo que abre la posibilidad de proponer otra 
organización del sentido de lo educativo y, por tanto, de los procesos y condiciones en los cuales se forman los sujetos de la educación.

Por ello, el trabajo de construir alternativas es un trabajo político pedagógico en el sentido de una intervención en el marco de un proyecto (Rodríguez, 2014). Una utopía que direcciona prácticas, un presente constituido por cierta perspectiva de futuro.

Lo alternativo no puede concebirse fuera de lo hegemónico, tiene como referente lo institucionalizado y puede surgir en el marco de las propias organizaciones o al margen de las mismas; se expresa a través de las expectativas y experiencias de los sujetos que reelaboran los saberes, producen, circulan y transmiten nuevas ideas que impactan de una u otra manera en la realidad. De ahí que los sujetos generen procesos alternativos que "se articulan en la sociedad con prácticas y saberes orientando transformaciones que de alguna manera marcan límites a lo instituido y lo reconfiguran" (Gómez, Hamui y Corenstein, 2013: 44).

Lejos estamos en este apartado de definir a los proyectos de ApS y a la propuesta de la cátedra de Educación, desarrollo local y territorial como una alternativa pedagógica. Sin embargo, entendemos que el enfoque del desarrollo territorial en diálogo con la pedagogía crítica nos ayuda a pensar otras maneras de abordar y de proponer los procesos de enseñanza-aprendizaje y de construir espacios educativos en diálogo con las problemáticas y demandas de los territorios. Encontramos en los proyectos de ApS algunas líneas para comenzar a trazar este recorrido.

Y tal como señala Tapia (2008b), encontramos en el ApS un espacio de articulación y de encuentro entre dos intencionalidades y culturas institucionales que a menudo se encuentran confrontadas en la vida de las escuelas, pero también de la universidad. Por un lado, la que tiene que ver prioritariamente con la calidad académica y, por otro, la que privilegia la responsabilidad y el compromiso social.

\section{Algunas reflexiones para una pedagogía desde los territorios}

A lo largo de este artículo buscamos reflexionar sobre la experiencia de tres años de una cátedra. Partimos del supuesto de que la reflexión sobre la práctica docente es un ejercicio de investigación-acción que se construye en el aula y junto con los/as estudiantes-docentes. Parte del sentido de realizar una sistematización tiene que ver con poder aprender de esa experiencia, con el objetivo de mejorar nuestras propias prácticas, compartir y socializar nuestros aprendizajes con experiencias similares y, finalmente, contribuir a la producción de nuevos conocimientos. En este sentido, presentamos tres dimensiones que emergieron de la experiencia de la cátedra y que contribuyen a producir conocimiento sobre nuestra propia práctica docente y sobre el rol de la universidad en la formación de educadores/as. 
En primer lugar, identificamos la distancia con que se presenta el concepto y el enfoque del desarrollo territorial para quienes se enmarcan dentro del campo educativo. Al asociar el concepto -y el ejercicio- de desarrollo territorial al crecimiento económico, este aparece como algo ajeno a las prácticas educativas de los y las estudiantes-docentes y de las instituciones en las que se desempeñan. Para trabajar sobre ello, y a partir de los aportes de la pedagogía crítica, realizamos el primer desplazamiento que consistió en desplegar una serie de estrategias pedagógicas para construir el aula en un espacio de diálogo y de articulación con los diferentes actores del territorio. $Y$ así poder reconstruir algunas experiencias de desarrollo territorial de Rafaela y la región. Al mismo tiempo, esto llevó a proponer -o al menos a imaginar- otro(s) tipo(s) de educación posible que comienzan a emerger en un segundo movimiento.

El segundo movimiento se da en la propuesta de comenzar a enmarcar a la institución educativa -y con ella a la universidad-como un actor más del territorio, capaz de establecer redes y articulaciones con otros actores. El redescubrir y reposicionar a la escuela pero también a la universidad en otro lugar produjo una ruptura en la reflexión de su práctica docente, pero también de la nuestra. Nos preguntamos: ¿A quiénes enseñamos?, ¿qué enseñamos? y ¿cómo la educación puede aportar al desarrollo de personas capaces de transformar los territorios?

Finalmente, la elaboración de los proyectos de Aprendizaje-Servicio contribuyó a materializar estas reflexiones. Como docentes encontramos en esta propuesta una posibilidad de articular el enfoque del desarrollo territorial con los procesos de enseñanzaaprendizaje $y$, al mismo tiempo, de entender a la universidad como un actor que debe estar en permanente diálogo con las demandas de los territorios. A su vez, los y las estudiantesdocentes de la licenciatura encontraron en esta propuesta una herramienta para proponer desde sus aulas. Analizar el proceso en desarrollo nos permitió reflexionar sobre la importancia y el desafío de nuestras universidades en la construcción de espacios de diálogos, de encuentros y acompañamiento desde una lógica de aprendizaje colectivo, horizontal y multidimensional.

A partir de nuestra propia experiencia, donde nos topamos con la dificultad actual de imaginar las prácticas de enseñanza-aprendizaje por fuera del aula, nos preguntamos si es posible proponer una pedagogía desde los territorios. Es decir, una pedagogía que no solo cuestione la centralidad de la ciencia (reconociendo otros saberes), de la educación bancaria (buscando constituir espacios emancipadores), sino que al mismo tiempo convierta a las instituciones educativas en verdaderos actores inmersos en los territorios y en donde se trabaje en una educación capaz de generar sujetos que se apropien de su territorio, lo comprendan, lo conozcan, se relacionen con los diferentes actores y construyan espacios de diálogo que se conviertan en espacios de aprendizajes y transformación. 


\section{Bibliografía}

Annette, J. (2005). Character, civic renewal and service learning for democratic citizenship in higher education. British Journal of Educational Studies, (3\}53, 326-340.

Alburquerque, F.; Ferraro, C. y Costamagna, P. (2008). Desarrollo económico local, descentralización y democracia: ideas para un cambio. San Martín: UNSAM Edita.

Arandia, M.; Alonso, M.J. y Martínez, I. (2010). La metodología dialógica en las aulas universitarias. Revista de Educación, 352, 309-329.

Arocena, J. (2013). El desarrollo local, una aproximación conceptual. + E: Revista de Extensión Universitaria, 3, 6-13.

Bednarz, S.; Chalkley, B.; Fletcher, S.; Hay, I.; Le Heron, E.; Mohan, A. y Trafford, J. (2008). Community Engagement for Student Learning in Geography. Journal of Geography in Higher Education, (1)32, 87-100.

Cecchi, N. (2006). Aprendizaje-servicio en Educación Superior. La experiencia latinoamericana. Seminario Internacional Responsabilidad Social Universitaria: Aprendizaje Servicio, 4.

Coraggio, J.L. (2004). La gente o el capital: desarrollo local y economía del trabajo. Buenos Aires: Editorial Abya Yala.

Costamagna, P. y Larrea, M. (2017). Actores facilitadores del desarrollo territorial. Una aproximación desde la construcción social. Deusto: Publicaciones de la Universidad de Deusto.

Costamagna, P. y Menardi, M. (2019). Repensando la relación entre universidades y municipios para el desarrollo territorial. En Procesos y metodologías participativas. Reflexiones y experiencias para la transformación social (pp. 389-410). Clacso-Udelar.

Costamagna, P. (2015). Política y formación en el desarrollo territorial. Aportes al enfoque pedagógico y a la investigación acción con casos de estudio en Argentina, Perú y País Vasco. Deusto: Publicaciones de la Universidad de Deusto.

(2020). Reflexiones y debates sobre el Desarrollo Territorial. Nuevas miradas frente a realidades complejas. Desarrollo y Territorio, 7, 7-16.

Elizalde, A.; Max-Neef, M. y Hopenhayn, M. (1986). Desarrollo a escala humana. Una opción para el futuro. Santiago de Chile: Centro de Alternativas de Desarrollo-CEPAUR y Fundación Dag Hammarskjold.

Escofet, A.; Folgueiras, P.; Luna, E. y Palou, B. (2016). Elaboración y validación de un cuestionario para la valoración de proyectos de aprendizaje-servicio. Revista mexicana de investigación educativa, (70)21, 929-949.

Freire, P. ([1965] 2008). La educación como práctica de la libertad. Buenos Aires: Siglo XXI. 
(2000). Cartas a Guinea Bissau. México: Siglo XXI.

Freire, P. y Macedo, D. (1987). Alfabetización. Lectura de la palabra y lectura de la realidad. Barcelona: Paidós.

Furco, A. (2003). Issues of definition and program diversity in the study of service-learning. In Billig, S. y Waterman, A. (eds.) Studying Service-Learning: Innovations in Education Research Methodology. Mahwah: Lawrence Erlbaum Associates.

Instituto de Capacitación y Estudios para el Desarrollo Local (Icedel) (2018). 4to. Censo Industrial de Rafaela 2018. $\mathrm{N}^{\circ} 4$ (p. 54). Icedel. Disponible en: http://icedel.rafaela.gob.ar/archivos/ORDICEdel/4to\%20CIndustrial.pdf (2020). Mapa Social de Rafaela. Resultados Relevamiento 2019. N² (p. 41). Icedel. Disponible en: http://icedel.rafaela.gob.ar/archivos/BV-PDF/mapacompleto.pdf

Jara, O. (1994). Para sistematizar experiencias: una propuesta teórica y práctica. San José de Costa Rica: Alforja.

Latorre, A. (2007). La investigación- acción. Conocer y cambiar la práctica educativa. Barcelona: Grao.

Madoery, O. (2006). La formación de agentes de desarrollo desde la perspectiva endógena. En Centro Latinoamérica de Administración para el Desarrollo. En: XI Congreso Internacional sobre la Reforma del Estado y de la Administración Pública, Guatemala.

Manzano, V. (2010). El modelo de aprendizaje-servicio y su potencial para la educación superior. Conferencia inaugural de las VI Jornadas de Docencia en Psicología, Universidad de Sevilla, 15 de junio.

Monclús, A. (1998). Pedagogía de la contradicción: Paulo Freire. Nuevos planteamientos en educación de adultos. Barcelona: Anthropos.

Peiretti, D. y Spinelli, E. (2016). Aprendizajes de un proceso de Sistematización de Experiencias de DT con alumnos y alumnas de la Maestría en DT (Año 2015). Rafaela: Instituto Praxis.

Puig, J. (2009). Aprendizaje servicio (ApS): educación y compromiso cívico. Barcelona: Grao.

Puig, J.; Batlle, R.; Bosch, C. y Palos, J. (2007). Aprendizaje servicio. Educar para la ciudadanía. Barcelona: Ministerio de Educación y Ciencia/Octaedro.

Puiggrós, A. y Gómez Sollano, M. (coords.) (2003). Alternativas pedagógicas. Sujetos y prospectiva de la educación latinoamericana. Buenos Aires: UBA, Instituto de Investigaciones en Ciencias de la Educación y UNAM, Facultad de Filosofía y Letras Educación, Miño y Dávila.

Reinders, H. (2010). The promotion of learning processes through service learning in higher education. Zeitschrift Fur Padagogik, 56(4), 531-547.

Rodríguez, M. (2014). El aprendizaje-servicio como estrategia metodológica en la universidad. Revista Complutense de Educación, (1)25, 95-113. 
Tapia, M.N. (2008a). Calidad académica y responsabilidad social: el aprendizaje servicio como puente entre dos culturas universitarias. En Martínez Martín, Miguel (2008). Aprendizaje servicio y responsabilidad social de las universidades. Barcelona: Octaedro. (2008b). Aprendizaje y servicio solidario. Buenos Aires: Ciudad Nueva.

Villar, A. (2007). Políticas municipales para el desarrollo económico-social: revisando el desarrollo local. Buenos Aires: Ediciones Ciccus.

Zemelman, H. (1987). Conocimiento y sujetos sociales: contribución al estudio del presente (Vol. 111). México: Colegio de México Centro de Estudios Sociológicos. 